\title{
Karyopherin binding interactions and nuclear import mechanism of nuclear pore complex protein $\mathrm{Tpr}$ Iris Ben-Efraim ${ }^{1}$, Phyllis D Frosst ${ }^{1,2}$ and Larry Gerace*1
}

Address: ${ }^{1}$ Department of Cell Biology, The Scripps Research Institute, La Jolla, CA 92037, USA and ${ }^{2}$ National Human Genome Research Institute, National Institutes of Health Bethesda, Maryland 20892, USA

Email: Iris Ben-Efraim - ibenefra@gmail.com; Phyllis D Frosst - Frosst@mail.nih.gov; Larry Gerace* - lgerace@scripps.edu

* Corresponding author

Published: 16 October 2009

BMC Cell Biology 2009, 10:74 doi:10.1 I86/147I-2I2I-10-74

Received: I June 2009

Accepted: 16 October 2009

This article is available from: http://www.biomedcentral.com/l47|-2/2I//0/74

(C) 2009 Ben-Efraim et al; licensee BioMed Central Ltd.

This is an Open Access article distributed under the terms of the Creative Commons Attribution License (http://creativecommons.org/licenses/by/2.0), which permits unrestricted use, distribution, and reproduction in any medium, provided the original work is properly cited.

\begin{abstract}
Background: $\mathrm{Tpr}$ is a large protein with an extended coiled-coil domain that is localized within the nuclear basket of the nuclear pore complex. Previous studies [I] involving antibody microinjection into mammalian cells suggested a role for Tpr in nuclear export of proteins via the CRMI export receptor. In addition, Tpr was found to co-immunoprecipitate with importins $\alpha$ and $\beta$ from Xenopus laevis egg extracts [2], although the function of this is unresolved. Yeast MlpIp and Mlp2p, which are homologous to vertebrate Tpr, have been implicated in mRNA surveillance to retain unspliced mRNAs in the nucleus[3,4]. To augment an understanding of the role of Tpr in nucleocytoplasmic trafficking, we explored the interactions of recombinant $\mathrm{Tpr}$ with the karyopherins CRMI, importin $\beta$ and importin $\alpha$ by solid phase binding assays. We also investigated the conditions required for nuclear import of Tpr using an in vitro assay.

Results: We found that Tpr binds strongly and specifically to importin $\alpha$, importin $\beta$, and a CRMI containing trimeric export complex, and that the binding sites for importins $\alpha$ and $\beta$ are distinct. We also determined that the nuclear import of Tpr is dependent on cytosolic factors and energy and is efficiently mediated by the importin $\alpha / \beta$ import pathway.

Conclusion: Based on the binding and nuclear import assays, we propose that Tpr is imported into the nucleus by the importin $\alpha / \beta$ heterodimer. In addition, we suggest that Tpr can serve as a nucleoporin binding site for importin $\beta$ during import of importin $\beta$ cargo complexes and/or importin $\beta$ recycling. Our finding that Tpr bound preferentially to CRMI in an export complex strengthens the notion that $\mathrm{Tpr}$ is involved in protein export.
\end{abstract}

\section{Background}

Molecules are transported between the cytoplasm and the nucleus through nuclear pore complexes (NPCs), massive proteinaceous structures that span the double membrane of the nuclear envelope (NE). Molecules smaller than 20-40 kDa in size can passively diffuse through the NPC. However most protein, and nucleic acid is transported by receptor and energy dependent mechanisms (reviewed in [5-8]).

Nucleocytoplasmic transport is mediated by shuttling transport receptors termed karyopherins or importins/ exportins (reviewed in $[5,7]$ ). In the extensively studied classical nuclear import pathway, cargoes carrying a basic 
amino acid-rich nuclear localization sequence (NLS) bind to the adaptor importin a, which in turn associates with the import receptor importin $\beta$ that mediates transport into the nucleus. A second class of import cargo directly binds to importin $\beta$ in the absence of an adaptor. In the classical nuclear export pathway, cargoes carrying a leucine-rich nuclear export signal (NES) bind to the exportin CRM1 together with RanGTP to be transported out of the nucleus.

The small GTPase Ran, which binds directly to both importins and exportins, plays a key role in determining the directionality of nuclear transport. The GTP-bound form of Ran is concentrated in the nucleus and the GDPbound form predominates in the cytoplasm, due to the nuclear localization of the Ran guanine nucleotide exchange factor RCC1 (RanGEF) and the cytoplasmic localization of the Ran GTPase- activating protein (RanGAP). The binding of RanGTP to karyopherins modulates the affinity of the receptors for cargo. When an importincargo complex encounters RanGTP in the nucleus, RanGTP promotes the dissociation of cargo from the receptor as well as dissociation of the importin from nucleoporins, and the importin-RanGTP complex is recycled back to the cytoplasm. The converse is true for exportins: intranuclear RanGTP promotes the binding of cargo to exportins, and when the RanGTP-containing export complex encounters RanGAP in the cytoplasm, GTP hydrolysis results in release of the cargo and regeneration of the free exportin [9-11].

The framework of the NPC consists of eight central spokes flanked by nuclear and cytoplasmic rings, forming a ringspoke assembly that surrounds a central transport channel. Extending outward from the ring-spoke assembly are 50-100-nm-long nuclear fibrils, which are joined in a basket-like structure ("nuclear basket"), and 35-50-nmlong cytoplasmic fibrils (reviewed in $[12,13]$ ). The NPC of both mammals and yeast comprise 30 different nucleoporins, which are present at integral multiples of 8 copies, consistent with the 8-fold rotational symmetry of the NPC framework. Within the NPC, nucleoporins are typically organized in distinct subcomplexes that are localized to specific regions of the NPC [13]. Approximately $1 / 3$ of the nucleoporins contain multiple copies of the FG (phenylalanine-glycine) di-amino acid repeat. These FG repeats are clustered in domains ( "FG domains") that are intrinsically unstructured. The FG domains appear to form the major diffusion barrier of the NPC $[14,15]$, and also serve as the key interaction sites for karyopherins during their transit through the NPC $[12,16]$.

In addition to undergoing reversible disassembly during mitosis in higher eukaryotes, NPCs are assembled throughout interphase in concert with NE growth [17]. Moreover many nucleoporins have intranuclear pools that appear to undergo dynamic exchange with NPC localized forms [18]. It is plausible that many if not most nucleoporins are imported into the nucleus by receptormediated pathways, but this process has not been studied in detail.

A conserved component of the NPC is the protein Tpr (for translocated promoter region) [19] and its homologs. Mammalian Tpr is a $267 \mathrm{kDa}$ structurally bipartite protein comprising 2,349 amino acids. Its N-terminal 1,600 residue domain associates in a dimer to form a parallel twostranded coiled-coil interrupted periodically along its length. The C-terminal domain comprising 800 amino acids is highly acidic and is predicted to be unstructured [20]. Tpr homologs have been characterized in Xenopus laevis [21], Saccharomyces cerevisiae (myosin-like proteins 1 and 2; Mlp1p and Mlp2p) [22], Drosophila melanogaster [23] and Arabidopsis thaliana [24]. In mammalian cells, Tpr is localized to the nucleoplasmic fibrils of the NPC $[1,25]$ and is suggested to act as the main architectural element of the nuclear basket [25]. Mammalian Tpr is tethered to the NPCs through interaction with Nup153 [26], whereas in yeast, Mlp1p and Mlp2p have been suggested to be anchored to the NPC by interactions with Nic96, or with Nup60 [27]. Numerous functions have been attributed to vertebrate Tpr and its yeast homologs Mlp1p and Mlp2p in addition to a role in NPC architecture. These involve mRNA export [27-30], nuclear protein export $[1,31]$, silent telomeric chromatin organization and telomere length control [32-34], spindle pole assembly in yeast [35], unspliced RNA retention $[4,36]$ and localization and stabilization of a desumoylating enzyme Ulp1 $[37,38]$. In addition, Drosophila Tpr has been linked to mitotic spindle organization and spindle checkpoint control [39].

In mammalian cells, classical nuclear protein import is not detectably affected in Tpr depleted cells $[1,40]$, whereas classical nuclear export is found to be significantly inhibited [1]. Yeast cells carrying a double deletion of Mlp1p and Mlp2p display markedly slower import of a model cargo [41], but protein export has not been examined. A biochemical study conducted with Xenopus Laevis egg extracts demonstrates that importin $\beta$ and importin $\alpha$ co-immunoprecipitate with Tpr [2]. Whether this interaction is direct or indirect was not investigated, and its biological significance remains unresolved. To gain further insight into the role of Tpr in nucleocytoplasmic trafficking of protein, we investigated the interaction of Tpr with the karyopherins involved in classical nuclear import and export using quantitative binding assays. Our findings indicate that Tpr binds specifically and with relatively high affinity to these nuclear transport receptors, and support the notion that Tpr provides a docking site for importin $\alpha / \beta$ and CRM1 in nuclear import and export. Furthermore, the results of our binding analysis together 
with in vitro nuclear import assays indicate that the nuclear import of Tpr is efficiently mediated by the classical importin $\alpha / \beta$ pathway.

\section{Results and Discussion Characterization of the binding of CRMI, importin $\beta$ and importin $\alpha$ to Tpr}

To investigate the interactions of Tpr with the karyopherins CRM1, importin $\beta$ and importin $\alpha$, we used quantitative solid phase binding analysis. To this end, we expressed recombinant full-length mammalian Tpr using a baculovirus system, and expressed the C-terminal domain of Tpr ("C-Tpr", residues 1626-2348) in bacteria. The expression and purification of these Tpr constructs was analyzed by SDS-PAGE (Fig. 1A). Full-length Tpr was found to migrate at its predicted molecular weight of $\sim 250$ $\mathrm{kDa}$, and immunoblotting with a mixture of antibodies raised against $\mathrm{C}$ and $\mathrm{N}$-terminal segments revealed an intact protein with no apparent proteolytic degradation (Fig. 1B). The migration of purified C-Tpr was in agreement with its calculated molecular weight of $112 \mathrm{kDa}$ (Fig. 1C).

We first analyzed the interactions of Tpr with CRM1 [1]. Because Tpr is localized to the nucleoplasmic side of the NPC, it could be a binding site for the trimeric CRM1 export complex at an early transport step. Alternatively, Tpr might have an indirect role in export related to receptor recycling or some other function. Since RanGTP and NES have been shown to cooperatively bind to CRM1 and to increase its affinity for nucleoporins that have been linked to export [42-44], we examined whether they would have an effect on the binding of CRM1 to Tpr. Tpr or C-Tpr were adsorbed to microtiter plates and the binding of CRM1 in the presence of NES peptide or NES peptide + RanGTP or absence of both was detected with primay antibodies raised against CRM1 and horseradish peroxidase-conjugated secondary antibodies. We found saturable binding of CRM1 to Tpr in the presence of an NES peptide or an NES peptide + RanGTP (Fig. 2A), although the apparent affinity of CRM1 for Tpr was significantly higher in the presence of NES + RanGTP $\left(K_{d}=83\right.$ $\mathrm{nM}$, Table 1$)$ as compared to NES alone $\left(K_{\mathrm{d}}=176 \mathrm{nM}\right.$, Table 1). In the absence of NES and RanGTP there was no saturable binding of CRM1 to Tpr in the concentration range analyzed. These results build on our previous finding that CRM1-mediated export is reduced considerably when Tpr is diminished or obstructed [1], and further suggest that Tpr is a docking site for the trimeric CRM1 nuclear export complex. Although Tpr depletion from cells was reported not to affect CRM1-mediated export in other studies [40], this might reflect differences in experimental approaches; e.g., cargo-receptor complex association with Tpr may not have been rate-limiting for export with the conditions used in the latter case [40].

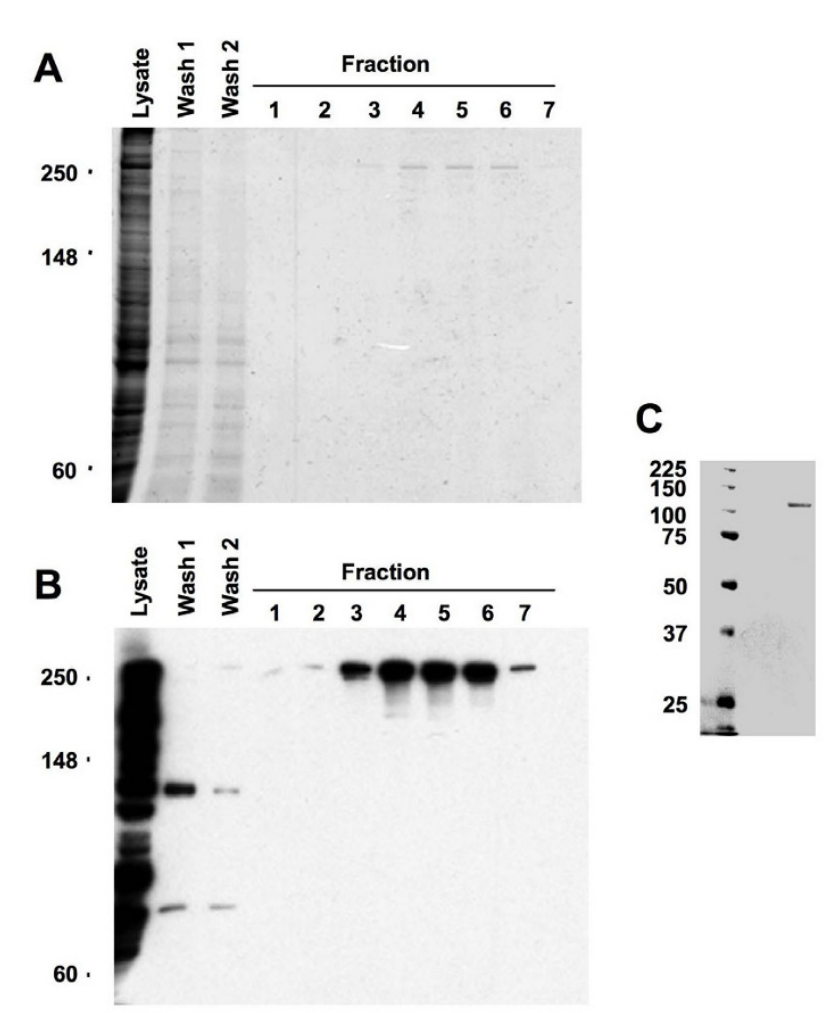

Figure I

Purification of recombinant Tpr from SF9 cells and C-Tpr (residues 1626-2348) from bacteria. Panel A: Coomassie stained SDS-PAGE (8\%) of a representative $6 \times$ His-Tpr purification. Shown are the cell lysate following centrifugation, washes $(800 \mathrm{mM} \mathrm{NaCl}, 20 \mathrm{mM}$ HEPES $\mathrm{pH} 7.5$, $10 \%$ glycerol, $15 \mathrm{mM}$ imidazole and protease inhibitors) to remove material non-specifically bound to the resin and seven elution fractions ( $100 \mathrm{mM} \mathrm{NaCl}, 20 \mathrm{mM}$ HEPES, I50 $\mathrm{mM}$ imidazole and $5 \mathrm{mM}$ beta-mercaptoethanol with protease inhibitors). Panel $B$ : Western analysis of the samples in Fig. I Panel A, probed with a combination of antibodies to the $\mathrm{N}$-terminus and the $\mathrm{C}$-terminus of Tpr. Panel C: Coomassie stained SDS-PAGE (8\%) of a representative purified CTpr(1626-2348).

In studies conducted in our laboratory and others, import of classical NLS cargoes was found to be unaffected in Tpr depleted cells $[1,40]$. However, a biochemical study conducted with Xenopus egg extracts demonstrated that importins $\alpha$ and $\beta$ co-immunoprecipitate with Tpr [2]. This study did not distinguish whether binding of importin $\beta$ and importin $\alpha$ to Tpr was direct or via an unidentified bridging factor. Importin $\beta$ binds directly to several FG repeat nucleoporins with apparent $K_{d}$ values between $9 \mathrm{nM}$ and $225 \mathrm{nM}$ depending on the nucleoporin [45], reflecting binding interactions of the importin $\beta$ import complex with the NPC during translocation. We examined whether Tpr was able to directly bind to importin $\beta$ 
Table I: Dissociation constants for Tpr interaction with: CRMI, importin $\beta$ or importin $\alpha$.

\begin{tabular}{ll}
\hline Recombinant Protein & $\boldsymbol{K}_{\mathrm{d}}$ apparent $(\mathbf{n M})$ \\
\hline CRMI & Too low to measure \\
CRMI+NES & $176 \pm 8(3)$ \\
CRMI+NES+RanGTP & $83 \pm 5(3)$ \\
Importin $\beta$ & $63 \pm 5(3)$ \\
Importin $\beta$ II78D & Too low to measure \\
Importin $\alpha$ & $21.5 \pm 1.5(3)$ \\
\hline
\end{tabular}

Data represent the mean \pm SD (number of repeats in parentheses) for the apparent dissociation constant

$K_{d}$ (see legend to Figs. I and 2).

and/or importin $\alpha$ in a similar affinity range. The binding between importin $\beta$ and Tpr (Fig. 2B) showed a saturable binding isotherm with a relatively high apparent affinity $\left(K_{\mathrm{d}}=50 \mathrm{nM}\right.$, Table 1$)$, demonstrating a direct interaction between the two proteins. Similar binding was found for C-Tpr (Fig. 2B), indicating that importin $\beta$ interacts with this region of Tpr.

Since the interaction of importin $\beta$ both with cargo and with nucleoporins is dissociated by RanGTP [46], as a specificity control we examined the effect of RanGMP-PNP and RanGDP- $\beta S$ (Ran bound to nonhydrolyzable analogs of GTP and GDP, respectively) on the binding of importin $\beta$ to Tpr (Fig. 3). Increasing concentrations of RanGMPPNP led to progressively less binding of importin $\beta$ to Tpr, and a 10 fold molar excess reduced binding to $42 \%$ of that observed in the absence of RanGMP-PNP (Fig. 3). RanGDP- $\beta S$ had no significant effect on the interaction of importin $\beta$ with Tpr. This indicates that RanGTP but not RanGDP decreases the binding of importin $\beta$ to Tpr, and argues for the biological specificity of the interaction we detected.

Importin $\beta$ is a superhelical solenoid of tandem HEAT repeats, which binds to nucleoporin FG repeats on its outer surface, and to RanGTP and importin $\alpha$ and other cargoes on its inner surface (reviewed in [7]). Since Tpr is a "cargo" that presumably needs to be imported into nuclei for interphase NPC assembly, the Tpr-importin $\beta$ interaction that we have detected could reflect a hypothetical cargo-like binding of Tpr to importin $\beta$ that is importin a-independent. Alternatively, it could reflect a possible FG repeat-like binding of importin $\beta$ to Tpr involved in importin $\beta$ trafficking. Indeed, Tpr contains 3 FG repeats in its C-terminal region that could provide such binding sites. To distinguish between these possibilities, we measured the affinity of the mutant importin $\beta$ I178D for Tpr. This mutant has a decreased affinity for FG repeats of nucleoporins due to disruption of a conserved hydrophobic binding pocket on the outer surface of importin $\beta$ [16]. However, the mutation has no effect on the binding to
A
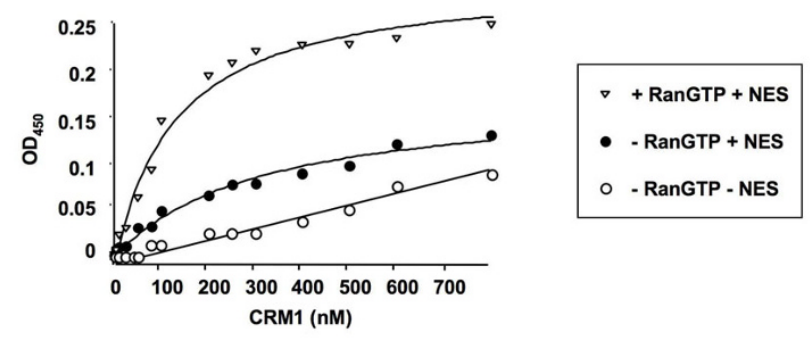

B
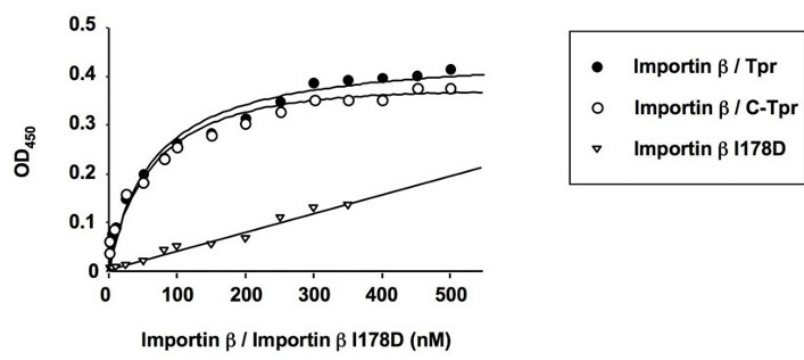

Figure 2

Characterization of the binding of CRMI or importin $\beta$ to Tpr or C-Tpr. Panel A: Binding was analyzed in the absence of RanGTP and NES (empty circles) or presence of RanGTP and NES (filled triangles) or absence of RanGTP and presence of NES (filled circles). Increasing eqimolar concentrations of CRMI, RanGTP and NES were incubated with $\mathrm{Tpr}$ and bound CRMI was quantified as described in materials and methods. Panel B: Binding to Tpr was analyzed with increasing concentrations of importin $\beta$ (filled circles) or importin $\beta$ II 78D (inverted triangles). Binding to C-Tpr was analyzed with increasing concentrations of importin $\beta$. The results are from duplicates of a single typical experiment. The standard deviation was $<5 \%$ of the mean. The data were fitted to the equation $\mathrm{B}(\mathrm{Y})=\mathrm{B}_{\max } \times \mathrm{Y} /\left(\mathrm{K}_{\mathrm{d}}+\mathrm{Y}\right)$ (using Kaleida Graph software) where $Y$ is the concentration of CRMI (Panel A) or importin $\beta /$ importin $\beta$ II 78D (Panel $B$ ) and $B$ is the amount of CRMI (Panel A) or importin $\beta /$ importin $\beta$ II 78D (Panel B) specifically bound. The correlation coefficients of the data to the fitted curves were $>0.99$.

either Ran or to various cargoes, including importin $\alpha$. We could not measure any specific binding of importin $\beta$ I178D for Tpr in the concentration range tested (Fig. 2B), indicating that the binding of Tpr involves the major FGrepeat binding pocket on the outer surface of importin $\beta$. This finding suggests that Tpr functions as a nucleoporin that provides a binding site for importin $\beta$ during import of importin $\beta$-cargo complexes or during recycling of cargo-free importin $\beta$, rather than an importin $\alpha$-independent import cargo.

We next measured the affinity of importin $\alpha$ for Tpr and C-Tpr (Fig. 4A). The results showed a saturable binding 


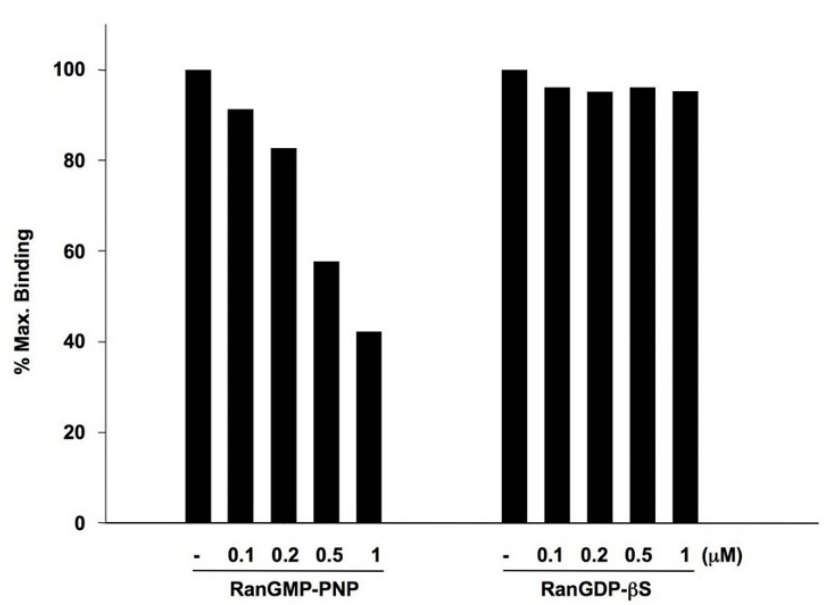

Figure 3

The binding of importin $\beta$ to Tpr in the solid phase assay is sensitive to RanGTP. The binding of $100 \mathrm{nM}$ importin $\beta$ to Tpr adsorbed to microtiter wells was analyzed in the absence, or in the presence of $0 . I, 0.2,0.5$ and I $\mu \mathrm{M}$ RanGMP-PNP or RanGDP $\beta S$, as indicated. The bound importin $\beta$ was quantified as described in materials and methods.

isotherm with a high apparent affinity $\left(\mathrm{K}_{\mathrm{d}}=21.5 \mathrm{nM}\right.$, Table 1) for both full length Tpr and C-Tpr, demonstrating a direct interaction between Tpr and importin $\alpha$. Similar values were recorded for the interaction between importin $\alpha$ and the SV40 T-antigen [47], a commonly studied cargo for importin $\alpha$. Since both importin $\alpha$ and importin $\beta$ are capable of directly binding to Tpr, we investigated whether they can bind to Tpr concurrently or whether they compete for each other's binding. For this analysis, we used an $\mathrm{N}$-terminal deletion mutant of importin $\alpha$ ( $\triangle$ IBB-importin $\alpha$ ) that lacks the importin $\beta$ binding domain, so that we could examine the binding of importin $\alpha$ to Tpr in the absence of its binding to importin $\beta$. We conducted competition-binding assays with importin $\beta$ kept at a constant concentration of $60 \mathrm{nM}$ ( $\sim$ the $\mathrm{K}_{\mathrm{d}}$ for Tpr) and the $\triangle \mathrm{IBB}$-importin $\alpha$ concentration increased from 0-600 nM. As shown in Fig. 4B, the $\triangle$ IBB-importin $\alpha$ does not diminish the binding of importin $\beta$ to Tpr at any concentration. Similar results were obtained with binding experiments that involved either importin $\alpha$ or $\triangle \mathrm{IBB}-$ importin $\alpha$ held at the constant concentration of $60 \mathrm{nM}$ and importin $\beta$ varied from 0-600 nM (data not shown). Together, these results indicate that importin $a$ and importin $\beta$ bind to different sites on Tpr.

\section{Nuclear Import of C-Tpr is mediated by the importin $\alpha$ l $\beta$ pathway}

Previous studies showed that the C-terminal domain of Tpr is responsible for its nuclear localization [41,28,48]. A minimal segment conferring nuclear localization is found
A

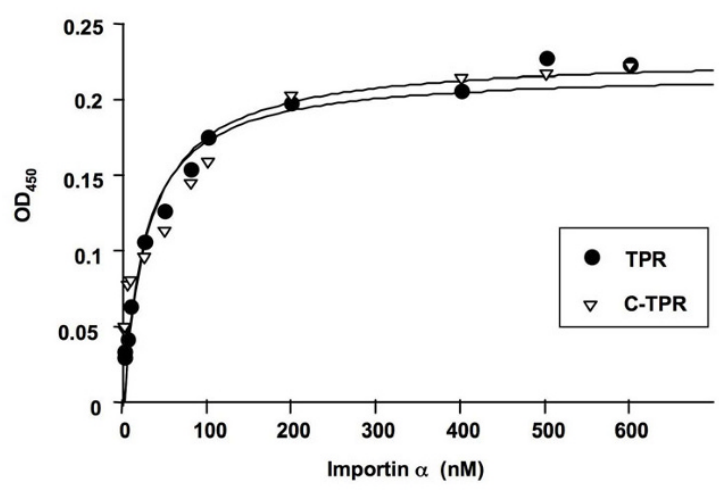

B

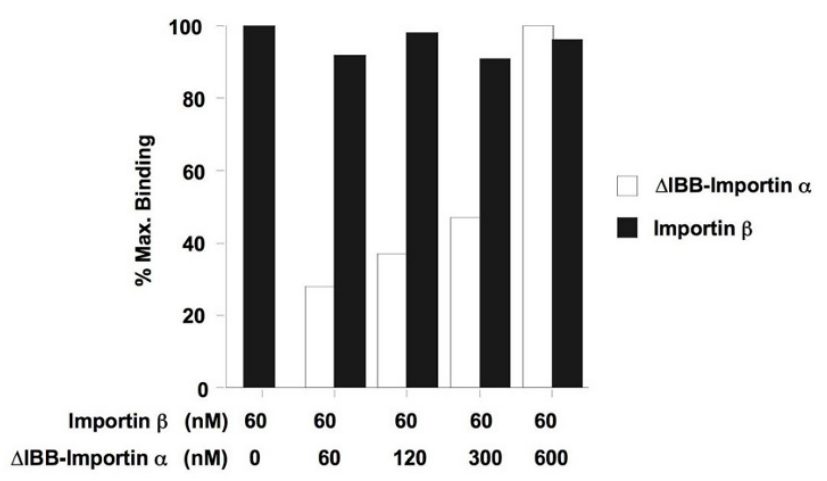

Figure 4

Characterization of the binding of $\triangle I B B$-importin $\alpha$ to Tpr and demonstration of different binding sites for $\triangle$ IBB-importin $\alpha$ and importin $\beta$ on Tpr. Panel A: Increasing concentrations of $\triangle \mathrm{IBB}$-importin $\alpha$ were incubated with immobilized Tpr or C-Tpr and the bound $\triangle \mathrm{IBB}$ importin $\alpha$ was quantified as described in materials and methods. The data were analyzed as described in Fig. I. Panel $\mathrm{B}$ : The binding of $60 \mathrm{nM}$ importin $\beta$ to Tpr pre-adsorbed to microtiter wells was analyzed in the absence of $\triangle \mathrm{IBB}$-importin $\alpha$ or in the presence of $60,120,300$ and $600 \mathrm{nM} \triangle \mathrm{IBB}$ importin $\alpha$, as indicated. Bound importin $\beta$ and $\Delta \mathrm{IBB}$-importin $\alpha$ were quantified as described in Materials and Methods.

in residues 1812-1867, and this contains a sequence resembling the bipartite basic amino acid NLS that is recognized by importin $\alpha$ [48]. These results, combined with our data showing that C-Tpr binds to importin $\alpha$ with a cargo-like affinity, suggest that the binding of Tpr to importin $\alpha$ could be important for Tpr import into the nucleus. We tested this hypothesis using digitonin-permeabilized NRK cells supplemented with exogenous cytosol to examine the nuclear import of the C-Tpr fragment. Nuclear import was visualized by the concentration of fluorescent cargo in the nucleus. NRK cells grow as flat, extensively spread cells that are 2-3 times the diameter of 
the nucleus (e.g. see Fig. 5, where fluorescent cargo is adsorbed to cytoplasmic structures). Full-length recombinant Tpr was unsuitable for this work, since it extensively aggregated during labeling and in the permeabilized cell assay (data not shown). Substantial nuclear accumulation of C-Tpr was detected when permeabilized cells were supplemented with cytosol and an energy-regenerating system at $30^{\circ} \mathrm{C}$ (Fig. 6). Nuclear import of $\mathrm{C}-\mathrm{Tpr}$ was inhibited when the reaction was carried out at $4{ }^{\circ} \mathrm{C}$ (Fig. 6) or when it was energy-depleted by treatment with hexokinase + glucose (Fig. 6). WGA, a lectin that binds to the NPC and that inhibits receptor-mediated transport, also inhibited the nuclear import of C-Tpr (Fig. 6). Collectively, these results indicate that nuclear accumulation of C-Tpr involves a temperature, energy and NPC-dependent process.

The potential involvement of importin $\alpha$ and importin $\beta$ in the nuclear import of C-Tpr was studied in digitoninpermeabilized cells reconstituted with recombinant transport factors (Fig. 5). We used the import of BSA-NLS of SV40 T-antigen, which is imported by the importin $\alpha / \beta$

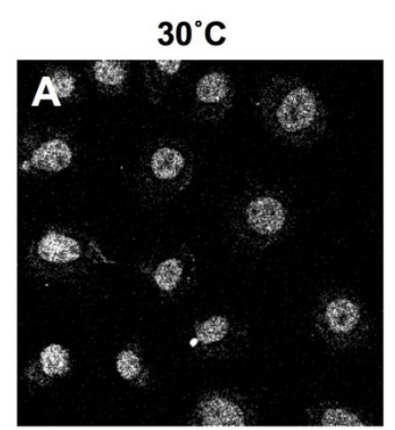

+WGA

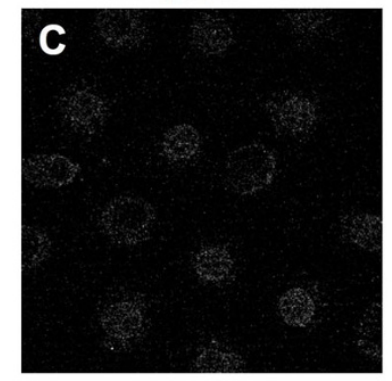

Figure 5

C-Tpr import into the nucleus is cytosol, temperature and energy dependent and is blocked by WGA. Digitonin-permeabilized NRK cells were incubated with 8 pmoles of Cy5-C-Tpr for $20 \mathrm{~min}$ at $30^{\circ} \mathrm{C}$ (panel A) or $4^{\circ} \mathrm{C}$ (panel B) in the presence of cytosol and an energy-regenerating system. Panel C: $8 \mu \mathrm{g}$ WGA was added. Panel D: the ATP regenerating system was replaced by an ATP-depleting system, $0.8885 \cup$ hexokinase + glucose. Samples were visualized by confocal microscopy. pathway, as a positive control. Nuclear import of C-Tpr was observed when the permeabilized cells were supplemented with exogenous recombinant importin $\alpha / \beta$ (Fig. 5 ) and was considerably diminished when either importin $\alpha$ or importin $\beta$ were omitted (Fig. 5), similar to the control cargo BSA-NLS, which requires both importins $\alpha$ and $\beta$ for nuclear import (Fig. 5). Nuclear import of fluorescently labeled C-Tpr was strongly diminished with a 10-fold molar excess of unlabeled C-Tpr (Fig. 5). Together these data support our main conclusion that a combination of importin $\alpha$ and importin $\beta$ are sufficient for efficient nuclear import of C-Tpr. This import pathway could be involved in Tpr assembly in the NPC during interphase, as well as at the end of mitosis. Since assembly of Tpr in the NPC at the end of mitosis follows that of almost all other nucleoporins [49], it is likely that Tpr enters the nucleus through largely if not completely intact NPC and thus it plausibly utilizes a receptor-mediated pathway to enter the nucleus.

\section{Conclusion}

Our quantitative binding studies showing that Tpr selectively binds to CRM1 present in a trimeric export complex supports the notion that Tpr plays a direct role in protein export [1]. Our binding studies further suggest that Tpr can provide a binding site for the import and/or recycling of importin $\beta$ complexes. Finally, the results presented here establish that nuclear import of Tpr can efficiently occur by the importin $\alpha / \beta$ import pathway.

\section{Methods \\ Plasmid Construction}

Recombinant baculovirus particles containing the mammalian Tpr cDNA were prepared using the Bac-to-Bac Baculovirus Expression System (Gibco BRL). The cDNA of Tpr was cloned into the Sal I site of the donor plasmid pFastBac HTc containing an N-terminal $6 \times$ His tag. The recombinant donor plasmid was transformed into DH10Bac Escherichia coli containing the viral backbone, where recombination generated a recombinant bacmid containing His-Tpr inserted into the viral backbone. Mammalian C-Tpr encompassing amino acids $1626-2348$ was constructed by inserting HindIII fragment into HindIII site of pET30c vector, and the construct was analyzed for correct orientation.

\section{Expression and Purification of Recombinant Proteins}

N-terminal His-tagged Tpr was expressed in SF9 cells at $27^{\circ} \mathrm{C}$ for 45 hours. Cells were lysed in RIPA buffer $(1 \%$ NP-40, 1\% sodium deoxycholate, 0.1\% SDS, $150 \mathrm{mM}$ $\mathrm{NaCl}, 10 \mathrm{mM}$ sodium phosphate, $\mathrm{pH} 7.2,2 \mathrm{mM}$ beta-mercaptoethanol and $2 \mathrm{mM}$ EDTA) with mammalian protease inhibitor cocktail (1:100, Sigma). Cell lysates were cleared at $14,000 \times \mathrm{g}$ and supernatants were purified on Talon affinity resin (Clontech). Only micorgram quanti- 


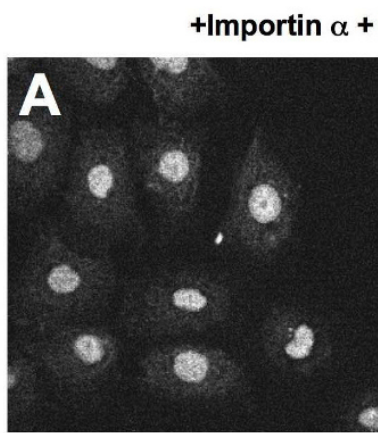

C-Tpr

+Importin $\alpha$ - Importin $\beta$

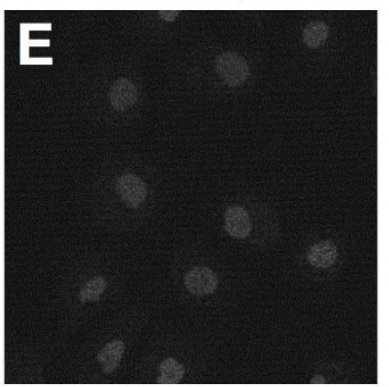

C-Tpr

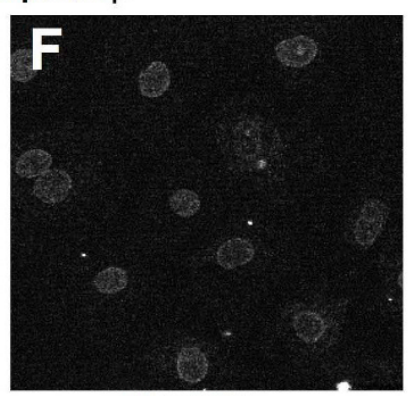

NLS-BSA

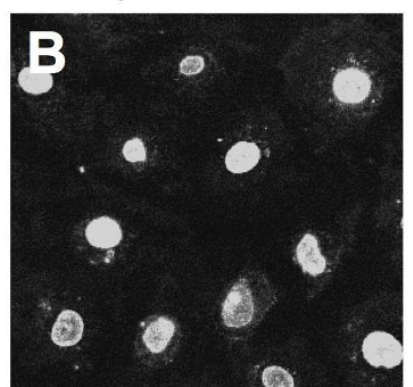

NLS-BSA

Figure 6

Role of importin $\alpha / \beta$ nuclear import pathway in the nuclear distribution of C-Tpr. Digitonin-permeabilized NRK cells were incubated with 8 pmoles Cy5-C-Tpr (Panels A, C, E, G) or 8 pmoles Cy3-BSA-NLS (coupled with a synthetic peptide comprising the NLS of SV40 T-antigen; Panels B, D, F, H) and nuclear import was conducted with recombinant factors as described in Materials and Methods. Panels A, B: nuclear import of Cy5-C-Tpr and Cy5-BSA-NLS performed at 30 ${ }^{\circ} \mathrm{C}$; panels C, D: importin $\alpha$ was omitted; panels E, F: importin $\beta$ was omitted; panels G, H: Cy5-C-Tpr and Cy3-BSA-NLS competed with I0fold molar excess of unlabeled cargo. Samples were visualized by confocal fluorescent microscopy.

ties of full-length Tpr could be obtained by this method. From Western blot analysis, we found that a significant amount of an immunoreactive band comigrating with purified full-length Tpr did not bind to the Talon resin, suggesting either that the His-tag was cleaved from this fraction, or that the His-tag was inaccessible, possibly because Tpr in the unbound fraction was present in small aggregates. Recombinant C-Tpr (comprising residues 1626-2348) was expressed in Escherichia coli BL21+ cells and grown at $37^{\circ} \mathrm{C}$ in $2 \times \mathrm{LB}$ medium to an $\mathrm{OD}_{600}$ of $0.5-$ 0.6. Expression was induced by the addition of $0.2 \mathrm{mM}$ IPTG for $3 \mathrm{~h}$ at $37^{\circ} \mathrm{C}$. Cells were resuspended in $50 \mathrm{mM}$ Tris buffer, pH 7.5 supplemented with $300 \mathrm{mM} \mathrm{NaCl}, 2$ $\mathrm{mM}$ DTT, $1 \mathrm{mg} / \mathrm{ml}$ lysozyme, $10 \mu \mathrm{g} / \mathrm{ml}$ DNase, and the protease inhibitor cocktail. The suspension was sonicated $(3 \times 20 \mathrm{~s})$, and centrifuged at $100,000 \times g$ for $30 \mathrm{~min}$. The supernatant was loaded on a Sepharose Q column, washed extensively with the lysis buffer containing 300 $\mathrm{mM} \mathrm{NaCl}$, and then eluted with a gradient of 300-530 $\mathrm{mM} \mathrm{NaCl}$ for 1 hour. Fractions containing C-Tpr were pooled; ammonium sulfate precipitated and run on a Superdex 75 column equilibrated in transport buffer.
Expression and purification of His-importin $\alpha, \Delta \mathrm{IBB}-$ importin a, importin $\beta$, His-S-tag-importin $\beta$, Ran, nuclear transport factor 2 (NTF2) and GST-M9 (a cargo of the nuclear import receptor, transportin) was performed as previously described [50]. These proteins were dialyzed into transport buffer [51] before use in the various experiments.

\section{Microtiter Plate Binding Assay}

Solid phase binding assays were carried out on microtiter plates (Maxisorp; Nunc) coated with $25 \mathrm{ng}$ of Tpr or CTpr. Assays were conducted as previously described [45]. Binding of His-importin $\alpha$ to Tpr or C-Tpr immobilized on microtiter plates was detected by an affinity-purified rabbit $\mathrm{pAb}$ raised against human His-importin $\alpha$. Binding of His-S-tag-importin $\beta$ to Tpr or to C-Tpr on microtiter plates was detected by an affinity-purified pAb raised against S-tag. Binding of CRM1 to Tpr in the presence or absence of a synthetic peptide comprising the NES derived from PKI (ELALKLAGLDIN) [52] and RanGTP was detected by affinity purified rabbit pAb raised against a Cterminal peptide derived from CRM1. Horseradish perox- 
idase-conjugated secondary antibodies were used for colorimetric detection (Pierce Chemical Co) using 3,3', 5,5'-tetramethylbenzidine as substrate (Calbiochem). The three components: CRM1, NES and RanGTP were used at equimolar concentrations in the binding experiments.

\section{Competition-binding assay}

Competition assays between $\triangle \mathrm{IBB}$-importin $\alpha$ and importin $\beta$ for binding to Tpr were carried out on microtiter plates (Maxisorp; Nunc) coated with $25 \mathrm{ng}$ Tpr. Different ratios of $\triangle \mathrm{IBB}$-importin a and importin $\beta$ were mixed together and incubated with immobilized Tpr. The experiments were carried out in duplicates. One repeat was probed with antibodies against S-tag to detect importin $\beta$ and the other repeat was probed with antibodies against $\Delta$ IBB-importin $\alpha$. Results are presented as percentage of highest level of binding.

\section{Nuclear Import Assay}

For analysis of nuclear import in digitonin-permeabilized adherent NRK cells, nuclear import in vitro assays, substrate visualization, and preparation of HeLa cytosol (by digitonin lysis) were carried out essentially as in [50]. 5 mg of C-Tpr was coupled with $\mathrm{Cy}^{\mathrm{TM}}$ ( 1 vial, Amersham Pharmacia Biotech. Inc.) for 30 minutes at room temperature and then separated from free dye by chromatography on a PD10 column (Amersham Pharmacia Biotech. Inc.) pre-equilibrated with transport buffer. Labeling of BSA with $\mathrm{Cy}^{\mathrm{TM}}$ was performed similarly. Conjugation of labeled BSA to a peptide derived from the NLS of SV40 Tantigen (CGGGPKKKRKVEDI) was performed as in [51]. Import reactions contained either $2.5 \mathrm{mg} / \mathrm{ml} \mathrm{HeLa} \mathrm{cytosol}$ or recombinant factors at the following concentrations: 100 nM His-importin $\alpha, 62.5 \mathrm{nM}$ importin $\beta, 450 \mathrm{n}$ Ran, $500 \mathrm{nM}$ NTF2. The import reaction mixture was supplemented with an energy-regenerating system and $1 \mathrm{mM}$ GTP.

\section{List of Abbreviations}

BSA: bovine serum albumin; CRM1: chromosome region maintenance 1; FG: phenylalanine glycine; Mlp1p: myosin-like protein 1; NE: nuclear envelope; NES: nuclear export signal; NLS: nuclear localization sequence; NPC: nuclear pore complex; NTF2: nuclear transport factor 2; pAb: polyclonal antibody; RanBP1: RanGTP binding protein; RanGAP1: RanGTPase activating protein; RanGEF: Ran guanine nucleotide exchange factor; RCC1, regulator of chromosome condensation 1; Tpr: translocated promoter region; WGA: wheat germ agglutinin.

\section{Authors' contributions}

IBE designed and performed the experiments, analyzed and interpreted data, and drafted the figures and manuscript. PDF prepared recombinant full-lengthTpr, performed preliminary versions of the binding assays and contributed intellectually to this development of the experiments. LG conceived and oversaw the conduct of the research and was involved in writing the manuscript. All authors read and approved the final manuscript.

\section{Acknowledgements}

We thank Dr. Ralph H. Kehlenbach for preparing the polyclonal antibodies against the C-terminus of CRMI. This work was supported by grant

GM4I955 to L.G.

\section{References}

I. Frosst P, Guan T, Subauste C, Hahn K, Gerace L: Tpr is localized within the nuclear basket of the pore complex and has a role in nuclear protein export. J Cell Biol 2002, I 56:617-630.

2. Shah S, Tugendreich S, Forbes D: Major binding sites for the nuclear import receptor are the internal nucleoporin Nup 153 and the adjacent nuclear filament protein Tpr. J Cell Biol 1998, | 41:3 | -49.

3. Vinciguerra P, Iglesias N, Camblong J, Zenklusen D, Stutz F: Perinuclear Mlp proteins downregulate gene expression in response to a defect in mRNA export. EMBO J 2005, 24:813-823.

4. Galy V, Gadal O, Fromont-Racine M, Romano A, Jacquier A, Nehrbass U: Nuclear retention of unspliced mRNAs in yeast is mediated by perinuclear MIp I. Cell 2004, I I 6:63-73.

5. Pemberton LF, Paschal BM: Mechanisms of receptor-mediated nuclear import and nuclear export. Traffic 2005, 6: 187-1 98.

6. Tran EJ, Wente SR: Dynamic Nuclear Pore Complexes: Life on the Edge. Cell 2006, I 25: I04I-1053.

7. Stewart M: Molecular mechanism of the nuclear protein import cycle. Nature Reviews Molecular Cell Biology 2007, 8: 195-208.

8. Mosammaparast N, Pemberton LF: Karyopherins: from nucleartransport mediators to nuclear-function regulators. Trends Cell Biol 2004, I 4:547-556.

9. Weis K: Nucleocytoplasmic transport throughout the cell cycle. Cell 2003, I I 2:44 I-45I.

10. Dasso M: The Ran GTPase: Theme and Variations. Curr Biol 2002, I 2:R502-R508.

II. Joseph J: Ran at a glance. J Cell Sci 2006, I 9:348I-3484.

I2. Fahrenkrog B, Koser J, Aebi U: The nuclear pore complex: a jack of all trades? TrendsBiochem Sci 2004, 29: I75-182.

13. Schwartz TU: Modularity within the architecture of the nuclear pore complex. Curr Opin Struc Biol 2005, I 5:22 I-226.

14. Denning DP, Patel SS, Uversky V, Fink AL, Rexach M: Disorder in the nuclear pore complex: the $F G$ repeat regions of nucleoporins are natively unfolded. Proc Natl Acad Sci USA 2003, I 00:2450-2455.

15. Denning DP, Rexach MF: Rapid evolution exposes the boundaries of domain structure and function in natively unfolded FG nucleoporins. Mol Cell Proteomics 2007, 6:272-282.

16. Bayliss R, Littlewood T, Stewart M: Structural basis for the interaction between FxFG nucleoporin repeats and importin $\beta$ in nuclear trafficking. Cell 2000, 102:99-108.

17. Antonin W, Ellenberg J, Dultz E: Nuclear pore complex assembly through the cell cycle: regulation and membrane organization. FEBS Lett 2008, 582:2004-16.

18. Dultz E, Zanin E, Wurzenberger C, Braun M, Rabut G, Sironi L, Ellenberg J: Systematic kinetic analysis of mitotic dis- and reassembly of the nuclear pore in living cells. J Cell Biol 2008, I 80:857-65.

19. Mitchell PJ, Cooper CS: Nucleotide sequence analysis of human Tpr cDNA clones. Oncogene 1992, 7:383-388.

20. Hase ME, Kuznetsov NV, Cordes VC: Amino acid substitutions of coiled-coil protein $\mathrm{Tpr}$ abrogate anchorage to the nuclear pore complex but not parallel, in-register homodimerization. Mol Biol Cell 200I, I 2:2433-2452.

21. Cordes VC, Reidenbach S, Rackwitz HR, Franke WW: Identification of protein $\mathrm{p270/Tpr}$ as a constitutive component of the nuclear pore complex-attached intranuclear filaments. J Cell Biol 1997, I 36:515-529.

22. Kolling R, Nguyen T, Chen EY, Botstein DA: New yeast gene with a myosin-like heptad repeat structure. Mol Gen Genet 1993, 237:359-369. 
23. Zimowska G, Aris JP, Paddy MR: A Drosophila Tpr protein homolog is localized to both in the extrachromosomal channel network and to nuclear pore complexes. J Cell Sci I997, I I 0:927-944.

24. Rose A, Patel S, Meier I: The plant nuclear envelope. Planta 2004, 21 8:327-336.

25. Krull S, Thyberg J, Bjorkroth B, Rackwitz HR, Cordes VC: Nucleoporins as components of the nuclear pore complex core structure and Tpr as the architectural element of the nuclear basket. Mol Biol Cell 2004, I 5:426I-4277.

26. Hase ME, Cordes VC: Direct interaction with nup I 53 mediates binding of Tpr to the pathway of the nuclear pore complex. Moll Cell Biol 2003, I 4: 1923-1940.

27. Kosova B, Pante N, Rollenhagen C, Podtelejnikov A, Mann M, Aebi U, Hurt E: Mlp2p, a component of nuclear pore attached intranuclear filaments, associates with nic96p. J Biol Chem 2000, 275:343-350.

28. Bangs P, Burke B, Powers C, Craig R, Purohit A, Doxsey S: Functional analysis of Tpr: identification of nuclear pore complex association and nuclear localization domains and a role in mRNA export. J Cell Biol 1998, I43:|80|-I8I2.

29. Green DM, Johnson CP, Hagan H, Corbett AH: The C-terminal domain of myosin-like protein I (Mlp Ip) is a docking site for heterogeneous nuclear ribonucleoproteins that are required for mRNA export. Proc Natl Acad Sci USA 2003, I 00:1010-1015.

30. Xu XM, Rose A, Muthuswamy S, Jeong SY, Venkatakrishnan S, Zhao $\mathrm{Q}$, Meier I: Nuclear pore anchor, the Arabidopsis Homolog of Tpr/MIp I/MIp2/Megator, Is Involved in mRNA Export and SUMO Homeostasis and Affects Diverse Aspects of Plant Development. Plant Cell 2007, I9:I537-I548.

31. Cornett J, Cao F, Wang CE, Ross CA, Bates GP, Li SH, Li JX: Polyglutamine expansion of huntingtin impairs its nuclear export. NatGenet 2007, 37:198-204.

32. Galy V, Olivo-Marin JC, Scherthan H, Doye V, Rascalou N, Nehrbass $\mathrm{U}$ : Nuclear pore complexes in the organization of silent telomeric chromatin. Nature 2000, 403:108-II2.

33. Hediger F, Dubrana K, Gasser SM: Myosin-like proteins I and 2 are not required for silencing or telomere anchoring, but act in the Tell pathway of telomere length control. J Struct Biol 2002, |40:79-9|.

34. Feuerbach F, Galy V, Trelles-Sticken E, Fromont-Racine M, Jacquier A, Gilson E, Olivo-Marin JC, Scherthan H, Nehrbass U: Nuclear architecture and spatial positioning help establish transcriptional states of telomeres in yeast. Nat Cell Biol 2000, 4:2 I4-22I.

35. Niepel M, Strambio-de-Castillia C, Fasolo J, Chait BT, Rout MP: The nuclear pore complex-associated protein, Mlp2p, binds to the yeast spindle pole body and promotes its efficient assembly. I Cell Biol 2005, I 70:225-235.

36. Casolari JM, Silver PA: Guardian at the gate: preventing unspliced pre-mRNA export. Trends Cell Biol 2004, 14:222-225.

37. Zhao X, Wu CY, Blobel G: Mlp-dependent anchorage and stabilization of a desumoylating enzyme is required to prevent clonal lethality. J Cell Biol 2004, I 67:605-6I I.

38. Palancade B, Liu X, Garcia-Rubio M, Aguilera A, Zhao X, Doye V: Nucleoporins Prevent DNA Damage Accumulation by Modulating Ulp I-dependent Sumoylation Processes. Mol Biol Cell 2007, I 8:29|2-2923

39. Lince-Faria M, Maffini S, Orr B, Ding $Y$, Florindo C, Sunkel CE, Tavares A, Johansen J, Johansen KM, Maiato H: Spatiotemporal control of mitosis by the conserved spindle matrix protein Megator. J Cell Biol 2009, I 84:647-57.

40. Shibata S, Matsuoka Y, Yoneda Y: Nucleocytoplasmic transport of proteins and poly $(A)+$ RNA in reconstituted Tpr-less nuclei in living mammalian cells. Genes Cells 2002, 7:42 I-434

4I. Strambio-de-Castillia C, Blobel G, Rout MP: Proteins connecting the nuclear pore complex with the nuclear interior. J Cell Biol 1999, 144:839-855.

42. Fornerod M, Van Deursen J, Van Baa S, Reynolds A, Davis D, Mutri KG, Fransen JG: The human homologue of yeast CRMI is in a dynamic subcomplex with CAN/Nup2 I 4 and a novel nuclear pore component Nup88. EMBO J 1997, 16:807-816.

43. Askjaer P, Bachi A, Wilm M, Bisschoff FR, Weeks DL, Ogniewski V, Ohno M, Nierhrs C, Kjems J, Mattaj I, Fornerod M: RanGTP-regulated interactions of CRMI with nucleoporins and a shuttling DEAD-box helicase. Mol Cell Biol 1999, 9:6276-6285.
44. Kehlenbach RH, Assheuer R, Kehlenbach A, Becker J, Gerace L: Stimulation of nuclear export and inhibition of nuclear import by a Ran mutant deficient in binding to Ran-binding protein I. J Biol Chem 2001, 276: |4524-|453|.

45. Ben-Efraim I, Gerace L: Gradient of Increasing Affinity of Importin $\beta$ for Nucleoporins along the Pathway of Nuclear Import. | Cell Biol 200I, | 52:4 | I-4 I8.

46. Rexach M, Blobel G: Protein import into nuclei: association and dissociation reactions involving transport substrate, transport factors, and nucleoporins. Cell 1995, 83:683-692.

47. Hu W, Jans DA: Efficiency of importin $\alpha / \beta$ mediated nuclear localization sequence recognition and nuclear import. J Biol Chem 1999, 274: I5820-I5827.

48. Cordes VC, Hase ME, Muller L: Molecular segments of protein Tpr that confer targeting and association with the nuclear pore complex. ECR 1998, 245:43-56.

49. Bodoor K, Shaikh S, Salina D, Raharjo WH, Bastos R: Sequential recruitment of NPC proteins to the nuclear periphery at the end of mitosis. Cell Sci 1999, I I 2:2253-2264.

50. Lyman SK, Guan T, Bednenko J, Wodrich H, Gerace L: Influence of cargo size on Ran and energy requirements for nuclear protein import. J Cell Biol 2002, 159:55-67.

51. Melchior F, Paschal B, Evans J, Gerace L: Inhibition of nuclear protein import by nonhydrolyzable analogues of GTP and identification of the small GTPase Ran/TC4 as an essential transport factor. I Cell Biol 1993, I 23: I649-I659.

52. Wen W, Meinkoth JL, Tsien RY, Taylor SS: Identification of a signal for rapid export of proteins from the nucleus. Cell 1995 82:463-473.
Publish with Biomed Central and every scientist can read your work free of charge

"BioMed Central will be the most significant development for disseminating the results of biomedical research in our lifetime. "

Sir Paul Nurse, Cancer Research UK

Your research papers will be:

- available free of charge to the entire biomedical community

- peer reviewed and published immediately upon acceptance

- cited in PubMed and archived on PubMed Central

- yours - you keep the copyright
BiolMedcentral 